\title{
A new result under weak and non-relativistic approximation from general theory of relativity
}

\section{Abhijit Samanta ( $\nabla$ abhijit.samanta@gmail.com )}

Jadavpur University

\section{Article}

Keywords: quantum physics, mechanics, matter waves, particle beams

Posted Date: February 4th, 2021

DOI: https://doi.org/10.21203/rs.3.rs-200345/v1

License: (c) (i) This work is licensed under a Creative Commons Attribution 4.0 International License. Read Full License 


\title{
A new result under weak and non-relativistic approximation from general theory of relativity
}

\author{
Abhijit Samanta \\ Nuclear and Particle Physics Research Centre \\ Department of Physics \\ Jadavpur University \\ Kolkata 700032, India \\ E-mail: abhijit.samanta@gmail.com \\ February 3, 2021
}

\begin{abstract}
We have derived a metric field equation in the locally inertial coordinate system from Einstein's field equation considering the energy density of the moving particle with the approximations that the force field under which the particle is moving is weak and the velocity of the particle is non-relativistic. We study the motion of different microscopic systems using this metric equation and compared the results with the experimentally measured values and we find that the results are identical.
\end{abstract}

\section{Motivation and background}

In the beginning of the previous century the Newtonian mechanics was advanced by two revolutionary theories: the general relativity (GR) and the Quantum Theory (QT). The phenomena related to the large masses are beautifully explained by GR, and QT predicts the results of experiments related to low masses. But, it is till unclear how GR and QT should be unified into a consistent theory. In conventional approach we treat gravity almost like any other forces and develop a quantum gravity theory, such as string theory. In the opposite approach, GR not only provides a unique and universal role for gravity among physical processes, but also, it is believed that straightforward quantization like other forces is not possible and it requires current framework of QT to be modified [1-4].

One of the most puzzling feature of QT is the measurement problem [5]. According to standard QT procedures of unitary evolution, entanglements have a tendency to spread through out the universe. But, in practical situation quantum state reduction is considered 
as though they become unentangled with the environment and leads us to a murky situation. There are some philosophical standpoint to accept this seeming contradiction: 1. Copenhagen view point of Neils Bohr, 2. environmental decoherence, 3. many-worlds viewpoint, and 4. conventional formulation of QT is provisional and some new theory is needed [6]. If we consider the opposite approach and modify QT assuming GR playing unique and universal role and predicted modification also allows test of unified theory of QT and GR far more achievable than probing the Planck scale where general relativistic effects are predicted in conventional quantum gravity theories.

Here, we study the motion of the particle moving under a general kind of weak field considering the energy density of moving particle. We first consider the field weak and stationary, then we consider the motion non-relativistic. Since all measurable quantities can only be expressed in inertial coordinate system, we transform the equation from general coordinate system to a locally inertial coordinate (LIC) systems using Einstein's equivalence principle. We compared the results with the experimentally measured values and we find that the results are identical. For an example, we study the motion of the electron in $\mathrm{H}$-atom.

\section{Calculation}

\subsection{Einstein's field equation under both weak and non-relativistic approximations}

The Einstein field equation under weak-field approximation [7-10],

$$
g_{\alpha \beta}=\eta_{\alpha \beta}+h_{\alpha \beta}, \quad\left|h_{\alpha \beta}\right|<<1
$$

reduces to the form

$$
\square \bar{h}_{\mu \nu}=2 \kappa \mathcal{T}_{\mu \nu}
$$

with Lorentz gauge condition

$$
\frac{\partial}{\partial x^{\mu}} \bar{h}^{\mu \nu}=0
$$

where,

$$
\bar{h}_{\mu \nu}=h_{\mu \nu}-\frac{1}{2} \eta_{\mu \nu} h, \quad h_{\mu \nu}=\bar{h}_{\mu \nu}-\frac{1}{2} \eta_{\mu \nu} \bar{h}, \quad h=-\bar{h}, \quad \square \equiv-\frac{\partial^{2}}{\partial t^{2}}+\nabla^{2} .
$$

$\mathcal{T}_{\mu \nu}$ is the energy-momentum density, and $h=\operatorname{Trace}\left[h_{\mu \nu}\right]$.

Now we consider the motion of the particle is non-relativistic, i.e., $\mathcal{T}_{00}>>\mathcal{T}_{i j}, \mathcal{T}_{0 i}$ and we neglect $\mathcal{T}_{i j}, \mathcal{T}_{i i}, \mathcal{T}_{i 0}$. Then Eq. 2 reduces to

$$
\square \bar{h}_{00}=-2 \kappa \mathcal{T}_{00}, \quad \square \bar{h}_{0 i}=0, \quad \square \bar{h}_{i j}=0 .
$$


In cases where $\mathcal{T}_{00}$ is not function of time, the above equations further reduce to

$$
\nabla^{2} \bar{h}_{00}=-2 \kappa \mathcal{T}_{00}, \quad \nabla^{2} \bar{h}_{0 i}=0, \quad \nabla^{2} \bar{h}_{i j}=0
$$

We consider the solutions of last two equations as

$$
\bar{h}_{i 0}=b_{i 0}, \quad \bar{h}_{i j}=b_{i j}
$$

where, $b_{i 0}, b_{i j}$ are arbitrary constants. We choose these constants other than zero. Though we neglect the variation of $\bar{h}_{i j}$ with space coordinates as we neglect $\mathcal{T}_{i j}$ compared to $\mathcal{T}_{00}$ to make our calculation simple; but, in practical cases $\bar{h}_{i j}$ may have small variations since $\mathcal{T}_{i j} \neq 0$, but these are negligibly very small compared to $\mathcal{T}_{00}$. We consider their average values neglecting the the variations. These small constants must have magnitude much smaller compared to $\bar{h}_{00}$. Now, the volume element of the space constructed by the metric $\bar{h}_{\mu \nu}$ remains 4-dimensional. Using Eqs 7 we see that Lorentz gauge condition (Eq. 3) is automatically satisfied.

\subsection{From general coordinate system to locally flat coordinate system}

The metric field Eq. 6 is expressed in general coordinate system. $\mathcal{T}_{\mu \nu}$ is energy-momentum density in general coordinate system. At any point $\mathrm{P}$ at $X=X_{0}$ in the curved space-time, we consider a locally flat coordinate (LFC) system $X^{\prime}$, which, in limited region of spacetime around $\mathrm{P}$, corresponds to a free falling, non-rotating over a short time interval. Now consider that, the whole space-time, in which the particle may have trajectories, is consist of a large number of such small coordinate patches (very small volume of space-time). which are locally flat ${ }^{1}$. Now at $X=X_{0}$ in the LFC system,

$$
\left.\frac{\partial g_{\mu \nu}}{\partial X^{\sigma}}\right|_{P\left(X=X_{0}\right)}=0
$$

and then using Eq. 1 and 4

$$
\left.\frac{\partial \bar{h}_{00}}{\partial X^{\sigma}}\right|_{P\left(X=X_{0}\right)}=0
$$

\footnotetext{
${ }^{1}$ If one chooses single inertial coordinate system with origin at infinity (as it is done in case of studies of planetary motion using Schwarzchild geometry of space-time), energy calculation at each point of the trajectory is possible, but energy density calculation is not possible since it varies rapidly with the change of space-time coordinates. Here, we find the metric of the space-time for a system using the energy density of the moving particle by solving Einstein's equation, while in planetary motion metric is determined from the free space solution of Einstein's equation using Schwarzchild geometry of space-time and energy density of the moving body was ignored.
} 
Now, we transform in LFC system from general coordinate system,

$$
\begin{array}{cc}
X \longrightarrow X^{\prime}, \quad \bar{h}_{\mu \nu}(X) \longrightarrow \bar{h}_{\mu \nu}^{\prime}\left(X^{\prime}\right), \\
X=X_{0}+X^{\prime}, \quad \bar{h}_{\mu \nu}(X)=\bar{h}_{\mu \nu}\left(X_{0}+X^{\prime}\right) .
\end{array}
$$

Now, using Taylor series expansion,

$$
\bar{h}_{\mu \nu}\left(X_{0}+X^{\prime}\right)=\left.\bar{h}_{\mu \nu}(X)\right|_{X=X_{0}}+\left.\frac{\partial \bar{h}_{\mu \nu}}{\partial X^{\rho}}\right|_{X=X_{0}} X^{\prime \rho}+\left.\frac{1}{2} \frac{\partial^{2} \bar{h}_{\mu \nu}}{\partial X^{\rho} \partial X^{\sigma}}\right|_{X=X_{0}} X^{\prime \rho} X^{\prime \sigma}
$$

Now, in LFC system $\bar{h}_{\mu \nu}\left(X_{0}+X^{\prime}\right)$ is replaced by $\bar{h}_{\mu \nu}^{\prime}\left(X^{\prime}\right)$ and then using Eq. 9 we find

$$
\bar{h}_{\mu \nu}^{\prime}\left(X^{\prime}\right)=\left.\bar{h}_{\mu \nu}(X)\right|_{X=X_{0}}+\left.\frac{1}{2} \frac{\partial^{2} \bar{h}_{\mu \nu}}{\partial X^{\rho} \partial X^{\sigma}}\right|_{X=X_{0}} X^{\prime \rho} X^{\prime \sigma}
$$

$\bar{h}_{\mu \nu}^{\prime}\left(X^{\prime}\right)$ can also be expanded in Taylor series,

$$
\bar{h}_{\mu \nu}^{\prime}\left(X^{\prime}\right)=\bar{h}_{\mu \nu}^{\prime}\left(X^{\prime}=0\right)+\left.\frac{\partial \bar{h}_{\mu \nu}^{\prime}}{\partial X^{\prime \rho}}\right|_{X^{\prime}=0} X^{\prime \rho}+\left.\frac{1}{2} \frac{\partial^{2} \bar{h}_{\mu \nu}^{\prime}}{\partial X^{\prime \rho} \partial X^{\prime \sigma}}\right|_{X^{\prime}=0} X^{\prime \rho} X^{\prime \sigma} .
$$

In LFC system, the second term of Eq. 14 are equal to zero and we find

$$
\bar{h}_{\mu \nu}^{\prime}\left(X^{\prime}\right)=\left.\bar{h}_{\mu \nu}^{\prime}(X)\right|_{X^{\prime}=0}+\left.\frac{1}{2} \frac{\partial^{2} \bar{h}_{\mu \nu}^{\prime}}{\partial X^{\prime \rho} \partial X^{\prime \sigma}}\right|_{X^{\prime}=0} X^{\prime \rho} X^{\prime \sigma}
$$

Comparing Eq. 13 and Eq. 15 we find the following identity:

$$
\bar{h}_{\mu \nu}\left(X=X_{0}\right)=\bar{h}_{\mu \nu}^{\prime}\left(X^{\prime}=0\right), \quad \nabla^{2} \bar{h}_{00}\left(X=X_{0}\right)=\nabla^{\prime 2} \bar{h}_{00}^{\prime}\left(X^{\prime}=0\right) .
$$

Here we argue that the region over which $\frac{\partial \bar{h}_{\mu \nu}^{\prime}}{\partial X^{\prime \rho}} \approx 0$ the identitities of Eq. 15 can be considered as the the variations of these quantities are small.

The transformation relation of the volume element (density of any quantity) under any coordinate transfromation, say, general coordinate system (described by metric element $\bar{h}_{\mu \nu}$ ) to LFC system (described by metric element $\bar{h}_{\mu \nu}^{\prime}$ ), is given by

$$
d^{4} X=\sqrt{\operatorname{det}\left[h_{\mu \nu}^{\prime}\left(X^{\prime}\right)\right]} d^{4} X^{\prime}, \quad \rho(X)=\rho^{\prime}\left(X^{\prime}\right) \sqrt{\left(\bar{h}_{\mu \nu}^{\prime}\left(X^{\prime}\right)\right)} .
$$

Now, the energy density $\mathcal{T}_{00}=T_{00}(X) \sqrt{\operatorname{det}\left[\bar{h}_{\mu \nu}(X)\right]}$ in general coordinate system, will be transformed to

$$
\begin{array}{r}
T_{00}^{\prime}\left(X^{\prime}\right) \sqrt{\operatorname{det}\left[\bar{h}_{\mu \nu}^{\prime}\left(X^{\prime}\right)\right]} \sqrt{\operatorname{det}\left[\bar{h}_{\mu \nu}(X)\right]}=T^{\prime}{ }_{00}\left(X^{\prime}\right) \sqrt{\mid \operatorname{det}\left[\bar{h}_{\mu \nu}^{\prime}\left(X^{\prime}\right)\right]} \sqrt{\operatorname{det}\left[\bar{h}_{\mu \nu}^{\prime}\left(X^{\prime}\right)\right]} \\
=T^{\prime}{ }_{00}\left(X^{\prime}\right) \operatorname{det}\left[\bar{h}_{\mu \nu}^{\prime}\left(X^{\prime}\right)\right]
\end{array}
$$


in LFC system. Then, first relation in Eq. 6 can be expressed in LFC system as

$$
-\nabla^{\prime 2} \bar{h}_{00}^{\prime}\left(X^{\prime}\right)=2 \kappa T^{\prime}{ }_{00}\left(X^{\prime}\right) \operatorname{det}\left[\bar{h}_{\mu \nu}^{\prime}\left(X^{\prime}\right)\right]
$$

From Eq. 7 (since $\bar{h}_{i j}^{\prime}$ also follows Eq. 6)

$$
\operatorname{det}\left[\bar{h}_{\mu \nu}^{\prime}\left(X^{\prime}\right)\right]=\bar{h}_{00}^{\prime}\left(X^{\prime}\right) b_{11} b_{22} b_{33}=b h_{00}^{\prime}\left(X^{\prime}\right) .
$$

Now, Eq. 20 becomes

$$
-\nabla^{\prime 2} \bar{h}_{00}^{\prime}\left(X^{\prime}\right)=2 b \kappa T^{\prime}{ }_{00}\left(X^{\prime}\right) \bar{h}_{00}^{\prime}\left(X^{\prime}\right)
$$

\subsection{Introducing arbitrary mass $m$ of the moving body in deter- mining $\bar{h}_{\mu \nu}^{\prime}\left(X^{\prime}\right)$}

If we know the energy $T^{\prime}{ }_{00}\left(X^{\prime}\right)$ of the particle at each point of a space-time, we can find the metric tensor $\bar{h}_{00}^{\prime}\left(X^{\prime}\right)$ of the space-time from Eq. 22 and hence the particle trajectory using this $\bar{h}_{00}^{\prime}\left(X^{\prime}\right)$ from geodesic equations. This particle trajectory is stationary since $\bar{h}_{00}^{\prime}$ is time independent. By definition of $g_{\mu \nu}$, the particle moving in the trajectory is considered to be of unit mass.

Now, if the energy at any point of the stationary trajectory of the particle of rest mass $m$ is $T_{00}^{\prime}$, then by definition of $g_{\mu \nu}$ and from Eq. 22, we find a particle of unit mass will follow the same stationary trajectory only when the energy is changed by $m$ times $T_{00}^{\prime}$. Of course we should keep all other conditions unchanged. See Eq. 34 for relation of $g_{00}$ and rest mass $m$ of the particle. This is true only when $T_{00}^{\prime}$ is a linear function of $m$. We will see later that for a moving particle under a weak potential, $T_{00}^{\prime}$ is proportional to $m$. So, for a particle of mass $m$ moving in a stationary trajectory and having a energy $T_{00}^{\prime}$ at $X^{\prime}$, the field equation will be

$$
-\frac{1}{2 m} \nabla^{\prime 2} h_{00}^{\prime}\left(X^{\prime}\right)=b \kappa T_{00}^{\prime}\left(X^{\prime}\right) \bar{h}_{00}^{\prime}\left(X^{\prime}\right)
$$

\subsection{Field equation for weak and stationary fields for a non-relativistic particle of negligible gravitational mass}

The LFC system can be approximated as locally inerial coordiante (LIC) system as

$$
\frac{\partial \bar{h}_{\mu \nu}^{\prime}}{\partial X^{\prime \rho}} \approx 0, \quad \bar{h}^{\prime} \approx 0
$$

The contributions to the total energy of the particle $T_{00}$ at any point of the trajectory are due to 1 . kinetic energy, 2. rest mass energy of the particle. Then, in LIC system, we can 
express Eq. 23 as

$$
-\frac{1}{2 m} \nabla^{\prime 2} \bar{h}_{00}^{\prime}\left(X^{\prime}\right)=b\left(\kappa^{\mathrm{kin}} E^{k i n}+4 \pi G m\right) \bar{h}_{00}^{\prime}\left(X^{\prime}\right) .
$$

The first term is for kinetic energy of the moving particle and it is proportional to $m$ as we requited in writing Eq. 23. ), The second term is for rest mass energy. The coefficient $\kappa^{\text {kin }}$ will be determined later. The energy density $E^{k i n}$ is generated due to the kinetic energy of the particle,

$$
E^{k i n}\left(X^{\prime}\right)=E^{\prime}-V^{\prime}\left(X^{\prime}\right)
$$

where, $V\left(X^{\prime}\right), E^{\prime}$ are the potential energy and total energy of the particle for a system. Then,

$$
-\frac{1}{2 m} \nabla^{\prime 2} \bar{h}_{00}^{\prime}\left(X^{\prime}\right)=b \bar{h}_{00}^{\prime}\left(X^{\prime}\right)\left[\kappa^{\mathrm{kin}}\left(E^{\prime}-V^{\prime}\left(X^{\prime}\right)\right)+4 \pi G m\right] .
$$

We rename the constant terms as, $k^{\text {kin }} E^{\prime}+4 \pi G m=k^{\text {kin }} E_{0}^{\prime}$. Denoting $k^{-1}=\frac{1}{b \kappa^{k i n}}$ we write Eq. 27 as

$$
-\frac{k^{-1}}{2 m} \nabla^{\prime 2} \bar{h}_{00}^{\prime}\left(X^{\prime}\right)+V^{\prime}\left(X^{\prime}\right) \bar{h}_{00}^{\prime}\left(X^{\prime}\right)=E_{0}^{\prime} \bar{h}_{00}^{\prime}\left(X^{\prime}\right)
$$

\section{Trajectory of the particle}

We consider that at any point of space-time the particle cannot be at rest, i.e., the particle can not exist if $T_{00}^{\prime}\left(X^{\prime}\right)=0$ (i.e., $E_{0}^{\prime}=0$ and $V^{\prime}\left(X^{\prime}\right)=0$ ). In this case the solution of Eq. 28 is

$$
\bar{h}_{00}^{\prime}=0
$$

We can use this solution as an boundary condition for solving Eq. 28. Then, there remains another arbitrary coefficient $b_{00}$ in the solution of $\bar{h}_{00}^{\prime} \neq 0$ of the Eq. 28. Here it can be determined from the boundary conditions, i.e., the initial velocity at initial position of the launching of the particle under the force field.

Once we know the metric $g_{\alpha \beta}$ at every point of the space-time for a system, we can find the trajectory of the motion. Using Eqs. 1, and 4

$$
g_{00}=\eta_{00}+b_{00} \bar{h}_{00}^{\prime}, \quad g_{11}=\eta_{11}+b_{11}, \quad g_{22}=\eta_{22}+b_{22}, \quad g_{33}=\eta_{33}+b_{33} .
$$

Neglecting $b_{11}, b_{22}, b_{33}$ as $b_{00} \bar{h}_{00}^{\prime}>>b_{i i}(i=1$ to 3$)$, the line element becomes

$$
d s^{2}=g_{00} d t^{2}-d r^{2}-r^{2}\left(d \theta^{2}+\sin ^{2} \theta d \phi^{2}\right) .
$$

And then using $d s^{2}=c^{2} d \tau^{2}$ one finds

$$
c^{2}=g_{00} \dot{t}^{2}-\dot{r}^{2}-r^{2}\left(\dot{\theta}^{2}+\sin ^{2} \theta \dot{\phi}^{2}\right)
$$


Now, Lagrangian (defined by $L=g_{\alpha \beta} \dot{x^{\alpha}} \dot{x^{\beta}}$ ) for the given system becomes

$$
L=g_{00} \dot{t}^{2}-\dot{r}^{2}-r^{2}\left(\dot{\theta}^{2}+\sin ^{2} \theta \dot{\phi}^{2}\right) .
$$

Then, one finds

$$
p_{0}=\frac{\partial L}{\partial \dot{t}}=g_{00} \dot{t}=\text { constant }=\frac{E_{0}^{\prime}+m c^{2}}{m c} .
$$

The geodesic equations are

$$
\begin{gathered}
\ddot{r}+\frac{1}{2} \frac{\partial g_{00}}{\partial r} \dot{t}^{2}-r\left(\dot{\theta}^{2}+\sin ^{2} \theta \dot{\phi}^{2}\right)=0, \\
\ddot{\theta}+\frac{1}{2 r^{2}} \frac{\partial g_{00}}{\partial \theta} \dot{t}^{2}+\frac{2 \dot{r} \dot{\theta}}{r}-\sin \theta \cos \theta \dot{\phi}^{2}=0, \\
\ddot{\phi}+\frac{1}{2 r^{2} \sin ^{2} \theta} \frac{\partial g_{00}}{\partial \phi} \dot{t}^{2}+2 \frac{\dot{r} \dot{\phi}}{r}+2 \frac{\dot{\theta} \dot{\phi}}{\tan \theta}=0 .
\end{gathered}
$$

Now, using the equations 35, 36 and 37 and the initial boundary conditions, the initial position and the velocity of the particle, one can find the trajectory of the particle. Here, one can choose a set of infinite number of boundary condition $\left.\dot{r}\right|_{\tau=0},\left.\dot{\theta}\right|_{\tau=0},\left.\dot{\phi}\right|_{\tau=0}$ as the arbitrary constant coefficient $b_{00}$ of the solution of $\bar{h}_{00}^{\prime}$ is fixed by Eq. 32 and 34 .

As an example, we study the motion of $1 s$ electron in H-atom. In solving Eq. 28 we use $k^{-1}=\hbar^{2}$ (where, $\hbar=h / 2 \pi, h$ is the Planck constant), we find the allowable values of $E_{0}^{\prime}$ form Eq. 28 are identical with the energy eigen values of the H-atom solution of Schrödinger equation. We also get satisfactory solution for other all microscopic systems if we consider $k^{-1}=\hbar^{2}$ as an universal constant as chosen in case of H-atom and consider $E_{0}^{\prime}$ as the energy of the system.

In Fig. 1 we show the trajectories of $1 s$ electron of H-atom for two different boundary conditions. It reveals the fact that the structure of $1 s$ orbit is different for different boundary conditions though the energy eigen-values of the metric equation Eq. 28 are same for all possible boundary conditions. It may lead to a general fact that, as in case of macroscopic world, the composite systems of same kind also (with the same eigen-state and eigen values of Eq. 28), are not exactly identical with each other as the trajectories of constituent particles are not same and it may shed light on the measurement problem in wave mechanics.

\subsection{Measurements and boundary conditions}

From Eqs. 34 we find that at any point if $g_{00}=1\left(\right.$ i.e., $\left.\bar{h}_{00}^{\prime}=0\right), \dot{t}=c$. This is not possible for a particle, the particle can not be at rest and particle can not exist at a point if $\bar{h}_{00}^{\prime}=0$. When $g_{00}$ differs largely from unity, $\dot{t}$ is low and from Eq. 32 we find that particle velocity is 

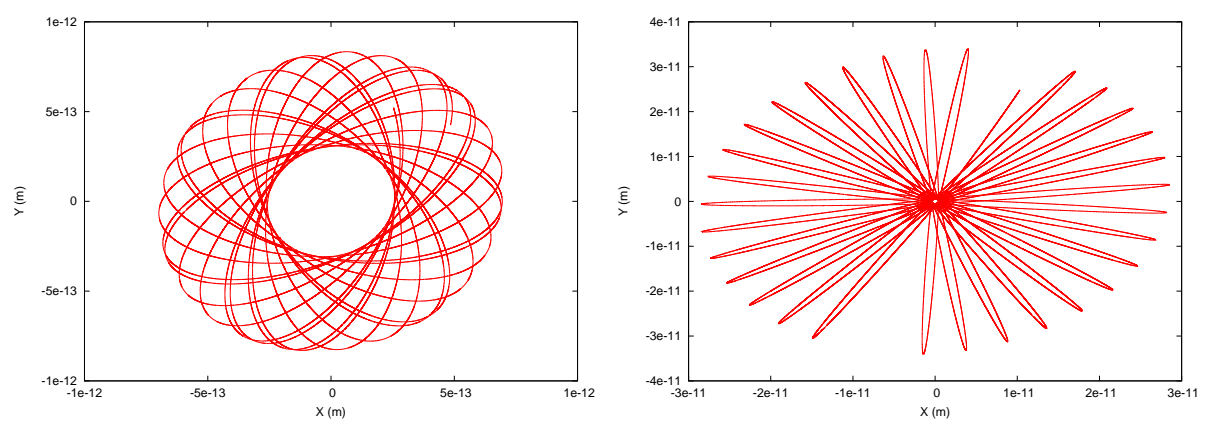

Figure 1: Two trajectories for $1 s$ electron in hydrogen atom in $X-Y$ plane for $b_{00}=-98.17$ (first plot ) and $-9.8 \times 10^{-5}$ (second plot) with typical initial positions and velocities. The end point positions and velocities are $\left(\tau, r_{\tau}, \theta_{\tau}, \phi_{\tau}, \dot{r}_{\tau}, \dot{\theta}_{\tau}, \dot{\phi}_{\tau}\right)=\left(2.98 \times 10^{-17}, \quad 5.87 \times\right.$ $\left.10^{-13}, \quad 1.42, \quad 114.216, \quad 1.725284 \times 10^{+6}, 1.67 \times 10^{+18}, 2.50 \times 10^{+18}\right)$ for first plot, and $\left(2.3 \times 10^{-15}, \quad 2.7 \times 10^{-11}, \quad 1.46, \quad 101.70, \quad 8.26134 \times 10^{+5}, \quad 7.95 \times 10^{+12} 1.16 \times 10^{+16}\right)$ for second plot, respectively in units of ( $\mathrm{m}, \operatorname{radian}, \operatorname{radian}, \mathrm{m} / \mathrm{s}, \operatorname{radian} / \mathrm{s}, \operatorname{radian} / \mathrm{s})$.

high and number of possible trajectories are also high since it suits with more wide ranges of boundary conditions as the ranges of the components of velocity are relatively wide. Finally we may arrive to the fact that at a point of space-time, if $\bar{h}_{00}^{\prime}$ is high, the possibility of finding the particle is high.

A range of initial boundary conditions for $\left.\dot{r}\right|_{\tau=0},\left.\dot{\theta}\right|_{\tau=0},\left.\dot{\phi}\right|_{\tau=0}$ are suited by the equations (see Eqs. 32, and 34). For each initial condition, we find a stationary orbit and one can get infinite number of such possible orbits. At present experimental situation in microscopic world, it is not possible to know the initial boundary conditions, say, in case of atoms, but one can predict the ranges of velocities and ranges of positions from the above equations. However, if we know the velocities at any point of space-time, we can predict the exact velocities or momentum at any other point of the space-time.

In the case when we do not know the initial boundary conditions, we can get a knowledge of position and momentum uncertainty from $\bar{h}^{\prime}\left(X^{\prime}\right)$. The particle can only exist at points where $\bar{h}^{\prime}\left(X^{\prime}\right) \neq 0$ and hence the width $\Delta X^{\prime}$ of this function determines the width of position uncertainty. Now if we Fourier transform this function $\bar{h}^{\prime}\left(X^{\prime}\right)$ we will get a new function and the width $\Delta P^{\prime}$ of this new function may determines the width of momentum uncertainty. From the properties of Fourier integral transform, these two widths can not be arbitrarily large or small and they follow the relation $\Delta X^{\prime} \cdot \Delta P^{\prime} \geq \hbar / 2$. Here, we choose $k^{-1}=\hbar^{2}$ as an universal constant.

For a free particle, we find from Eq. 28 that the metric $h_{00}^{\prime}\left(X^{\prime}\right)$ is oscillatory and then from above discussion it is clear that the particle trajectory is not a straight line and it is an undulating line in the space-time. We may use this fact to explain the the diffraction of 
the microscopic particles.

\section{Conclusion and future outlook}

We have derived a differential equation with the perturbed part $\bar{h}_{00}^{\prime}\left(X^{\prime}\right)$ of the metric under weak and non-relativistic approximations in locally inertial coordinate system. We have considered the energy density of the moving particle in finding the differential equation of the metric field. Then we solve the equation for different microscopic systems and the results are identical with experimentally measured values. We have shown the stationary electron trajectories in $\mathrm{H}$-atom for different boundary conditions as examples and then we discussed on the results of the measurable quantities.

\section{Appendix A Calculation of energy density for a point particle in general coordinate system}

For a system of point particles the special-relativistic energy-momentum tensor at $x$ is

$$
T^{\alpha \beta}=\sum_{n} m_{n} \int \frac{d x_{n}^{\alpha}}{d \tau} d x_{n}^{\beta} \delta^{4}\left(x-x_{n}\right)
$$

The four dimensional delta function in general coordinate system is defined by

$$
\int d^{4} x \Phi(x) \delta^{4}\left(x-x_{n}\right)=\Phi(y)
$$

Since $g^{1 / 2} d^{4} x$ is scalar, $g^{-1 / 2} d^{4}(x-y)$ must be a scalar $\left(g \equiv-\operatorname{det}\left[g_{\alpha \beta}\right]\right)$. Thus the contra-variant tensor that agrees Eq. 38 in Minkowski space (in absence of any source which produces curvature in space) becomes in general coordinate system

$$
T^{\mu \nu}=g^{-1 / 2} \sum_{n} m_{n} \int \frac{d x_{n}^{\mu}}{d \tau} d x_{n}^{\nu} \delta^{4}\left(x-x_{n}\right)
$$

in general coordinate system.

The energy-momentum tensor for the system becomes

$$
\sum_{n} m_{n} \dot{x_{n}^{\mu}} \dot{x_{n}^{\nu}}=\int d^{4} x T^{\mu \nu} g^{1 / 2}
$$

where, $T^{\mu \nu} g^{1 / 2}$ is the energy-momentum density in general coordinate system.

Now, in the space-time described by the metric $\bar{h}_{\mu \nu}$ Eq. 41 becomes

$$
\sum_{n} m_{n} \dot{x_{n}^{\mu}} \dot{x_{n}^{\nu}}=\int d^{4} x \sqrt{\operatorname{det}\left[\bar{h}_{\mu \nu}\right]} T^{\alpha \beta} .
$$


Acknowledgement: The author wishes to thank Dr. Abhik Sanyal, Jangipur College, West Bengal, India for discussion on critical points on the derivation and for the valuable comments for improvements of the manuscript. The work was supported by RUSA-2.0 project, Jadavpur University. The computer cluster facility of department of physics, Jadavpur University is also acknowledged.

\section{References}

[1] R. Howl, R. Penrose and I. Fuentes, New J. Phys. 21, no.4, 043047 (2019) doi:10.1088/1367-2630/ab104a [arXiv:1812.04630 [quant-ph]].

[2] R. Penrose, Proc. Roy. Soc. Lond. A 284, 159 (1965) doi:10.1098/rspa.1965.0058

[3] R. Penrose, Gen. Rel. Grav. 28, 581-600 (1996) doi:10.1007/BF02105068

[4] R. Penrose, J. Phys. Conf. Ser. 174, $012001 \quad$ (2009) doi:10.1088/1742$6596 / 174 / 1 / 012001$

[5] For complete references see, e.g., book by Cohen-Tannoudji, Claude and Diu, Bernard and Lalo, Franck (1977), Quantum mechanics, Wiley, New York, NY; and also, E. Schrödinger, Phys. Rev. 28, no. 6, 1049-1070, (1926), doi:10.1103/PhysRev.28.1049. Louis de Broglie, The London, Edinburgh, and Dublin Philosophical Magazine and Journal of Science, 47, 278, 446-458, (1924), Taylor \& Francis, doi:10.1080/14786442408634378. M. Born, Z. Phys. 26, 379 (1924); (2), vol. 2, p. 61.

[6] R. Penrose, Phil. Trans. Roy. Soc. Lond. A 356, 1927-1938 (1998)

[7] A. Einstein, Annalen Phys. 49, no.7, 769-822 (1916), doi:10.1002/andp.200590044

[8] For complete reference see, e.g., book by S. Weinberg, (1972). Gravitation and Cosmology: Principles and Applications of the General Theory of Relativity. Wiley. ISBN 978-0-471-92567-5

[9] M. P. Hobson, G. P. Efstathiou and A. N. Lasenby (2006), "General relativity: An introduction for physicists," Cambridge University Press.

[10] J. B. Hartle (2006), “An introduction to Einstein's general relativity," Pearson Education. 
Figures
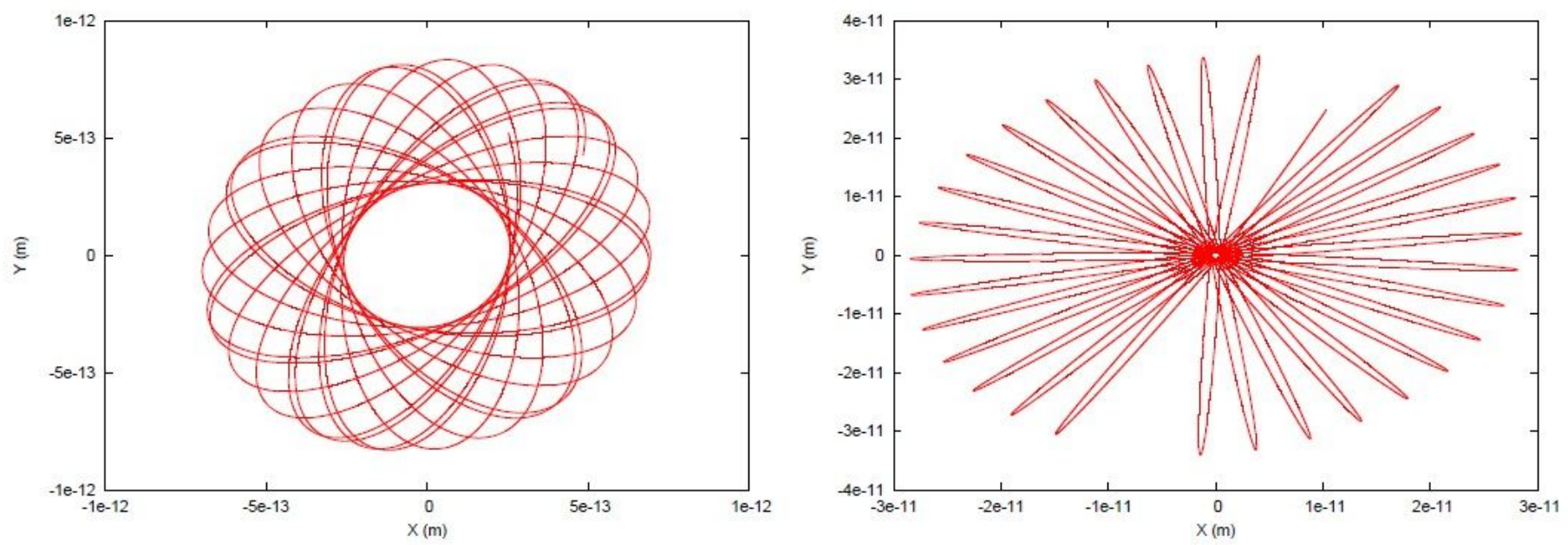

Figure 1

Two trajectories for $1 \mathrm{~s}$ electron in hydrogen atom in $X-Y$ plane (see Manuscript file for full figure legend) 